\title{
DESIGN AND IMPLEMENTATION OF A DVB ON-BOARD MULTI-CARRIER DEMODULATOR
}

\author{
J. Sala-Álvarez, A. Pagès-Zamora, S.Calvo, J.Prat ${ }^{(1)}$ \\ Department of Signal Theory and Communications, Universitat Politècnica de Catalunya \\ c/Gran Capità, s/n, Mòdul D5, 08034 Barcelona, SPAIN \\ Tel: +34-3-4016440, Fax:+34-3-4016447. \\ E-mail: alvarez@gps.tsc.upc.es \\ (I) Alcatel Espacio, Parque Tecnológico Tres Cantos, Madrid
}

\begin{abstract}
A description of the signal processing stage of an on-board integrated VLSI multi-carrier demodulator at the demultiplexing level is presented in this paper, along with a description of the optimization procedure that has been developed for the signal processing functions. The varying adjacent carrier interference and channel noise distribution are modeled to provide the best performing demultiplexing scheme under the given carrier distribution with mininum complexity.
\end{abstract}

\section{INTRODUCTION}

Space Digital Video Eroadcasting Systems are evolving toward the DVB (Digital Video Broadcasting) Standard based on MPEG2. An increasingly larger amount of processing is being moved toward the space segment, so that complex regenerative payloads shall have to be carried by the forthcoming satellite generation. In this system, several ground stations access the satellite on the up-link in a multi-frequency TDMA scheme. Carriers are demultiplexed, demodulated and merged in a single DVB stream at $33 \mathrm{Mbps}$ on the down-link. On-board systems demand very efficient techniques from system designers. Therefore, the filtering stages of the digital demultiplexer have to be optimized according to some complexity criterion to minimize power consumption, gate count and implementation losses without detriment to performance. A special criterion that takes into account the structure of the (interfering) adjacent carriers is developed in this paper to derive suitable decimation filters for the demultiplexing function. The criterion optimizes jointly the filter response in the pass-, transition and stop-bands for a given number of coefficients as the complexity of filtering is exponential in the filter length. The large bandwidth that the demultiplexer must process is very demanding in terms of processing speed. Hence, small efficient filters are necessary.

A system overview is presented in section 2: System Description. Section 3, Filter Design, deals specifically with the optimization of filters for VLSI integration. Simulation results are shown in Section 4 and conclusions are drawn in Section 5.

\section{SYSTEM DESCRIPTION}

The architecture of the digital on-board demultiplexer ${ }^{1}$ shall have to deliver any carrier combination of those allowed (see Fig. 2.1

1 This research work has been partially supported by the National Research Plar: of Spain (CYCIT): TIC95-1022-C05-01 and 2.2) of the following signaling rates: $R_{s}, 2 R_{s}, 3 R_{s}$ and $4 R_{s}$, with $R_{s}$ the lowest signaling rate. Each carrier is QPSK modulated with a square root raised cosine pulse (roll-off 0.35 ). Two possible frequency plans have been tailored to facilitate the demultiplexing scheme, where the separation with adjacent carriers is $1.5 R_{s}$. In the final architecture, both frequency plans depicted in Fig. 2.1 and Fig. 2.2 are processed by two independent demultiplexers that can be internally configured to deal with either of them. The overall bandwidth ( $36 \mathrm{MHz}$ ) can contain up to 18 small carrier at the $R_{s}$ signaling rate. The sampling scheme is IF sampling at fs $=36 \mathrm{Rs}(45 \mathrm{MHz})$.

Both frequency plans have been devised to contain the four possible mentioned signaling rates with two constraints: (a) that very simple frequency shifting operations should be carried and (b) that the output sampling rate of each carrier should be the same (in samples per symbol) for all rates. These two constraints have led to the construction of two frequency plans and the design of the demodulators at 3 samples per symbol.

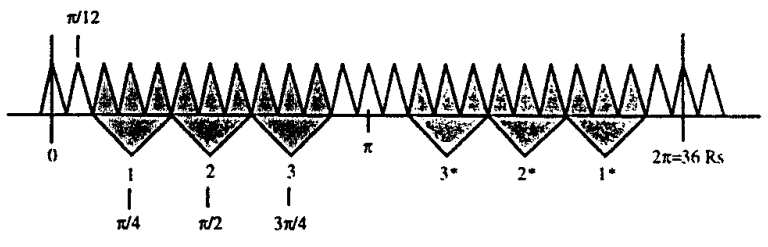

Figure 2.1. Frequency Plan No. 1 for 2.1 and $6.3 \mathrm{Mbps}$ carriers (spectrum of the input real signal) IF sampling is performed at $\mathrm{fs}=36 \mathrm{Rs}$. The carrier allocation displays three-fold granularity. The possible modulated carriers are shown shaded.

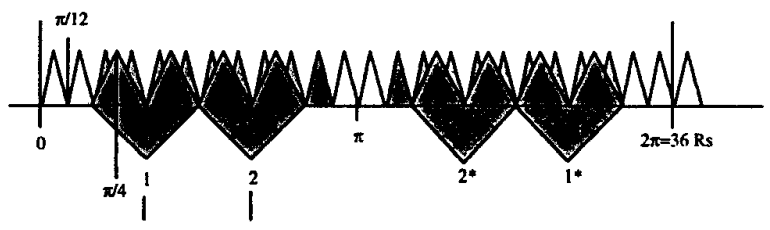

Figure 2.2. Frequency Plan No. 2 for $2.1,4.2$ and 8.4 Mbps carriers (spectrum of the input real signal). IF sampling is performed at $\mathrm{fs}=36 \mathrm{Rs}$. The carrier

and TIC96-0500-C10-01 and the Catalonian Regional Government (CIRIT): 1996SGR-00096 
allocation displays two-fold granularity. One extra Rs carrier is included to optimize band re-use.

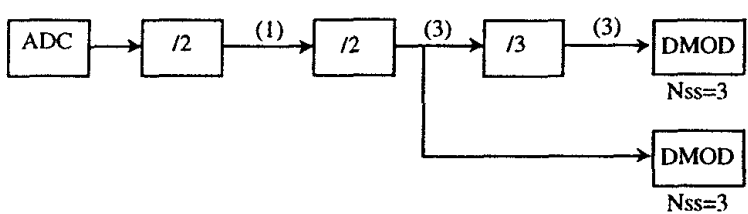

Figure 2.3. Structure of the filtering and decimation stages for Frequency Plan No. 2 that deliver each carrier to the bank of demodulators at 3 samples per symbol.

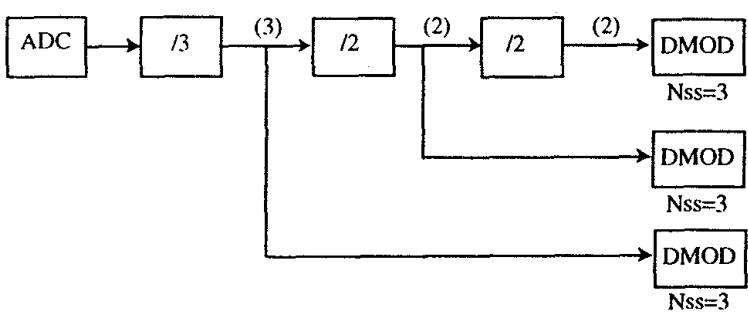

Figure 2.4. Structure of the filtering and decimation stages for Frequency Plan No. 2 that deliver each carrier to the bank of demodulators at 3 samples per symbol.

The inner architecture of the blocks denoted ' $/ 2$ ' (decimation by two) and ' $/ 3$ ' (decimation by three) in Fig. 2.3 and 2.4 consists of intercommunicating polyphase processors in a tree scheme. The design of the optimum subfilters of the 6-bin ('/3') and 4-bin ('/2') processors is carried out in Section 3: Filter Design.

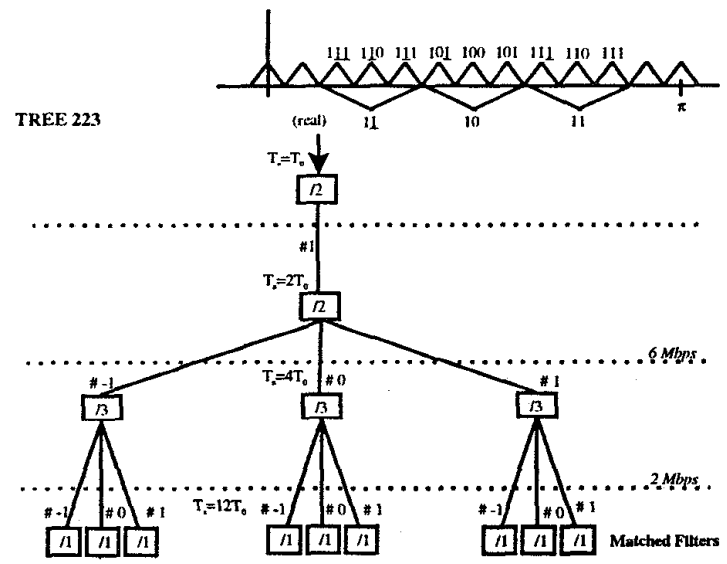

Figure 2.5. Carrier and processing time allocation to each of the filtering and decimation processors for Frequency Plan No. 1.

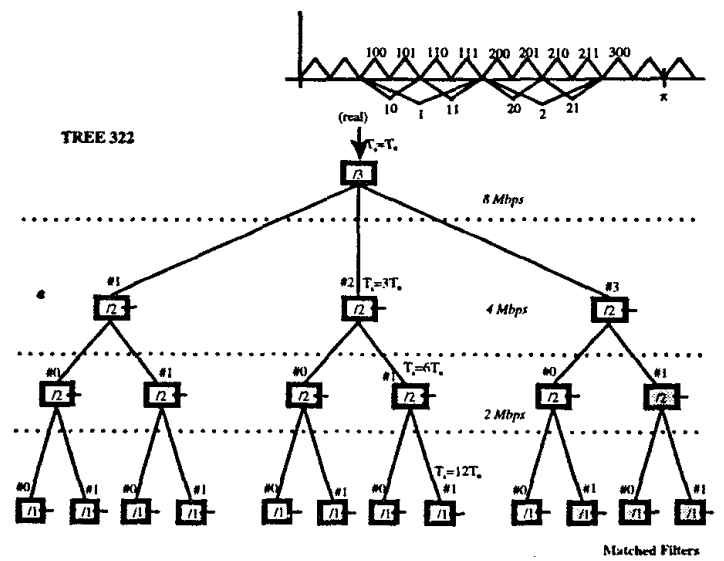

Figure 2.6. Carrier and processing time allocation to each of the filtering and decimation processors for Frequency Plan No. 1. The non-operational processors in the tree appear shaded.

\section{FILTER DESIGN}

In this section, the design of the filters $\left\{h_{i}(n), i=0, \ldots, 5\right\}$ of the de/multiplexing trees is approached. To that purpose a Minimum Mean Squared Error (MMSE) criterion is considered in order to improve the trade-off between the final EbNo (bit energy to noise spectral density ratio) and the hardware implementation cost of the filters, which increases exponentially with the filter length. The generic design problem is shown in Fig. 3.1 with $x_{B P}(n)$ the input signal (where BP stands for band-pass signal), $y_{B P}(n)$ the output signal, $d_{B P}(n)$ the reference signal and $e_{B P}(n)$ the error or difference signal. Note also that the a decimation rate $\mathrm{N}: 1$ is included in the design scheme and that a central frequency, $\omega_{0}$, for the band-pass filter is considered.

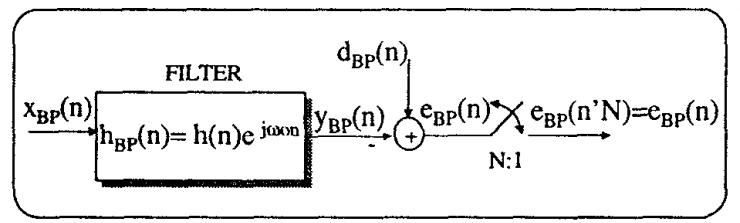

Figure 3.1. The generic filter design problem.

Since for all the filters of the Fig.2.5-2.6 $\omega_{0}$ equals the central frequency of the carrier of interest, the filter design problem can be stated in terms of the equivalent low-pass (ELP) signals, denoted without the subscript $B P$. Thus, the input signal $x(n)$ consists of the carrier of interest $d(n)$, all the interfering carriers gathered in $\mathrm{i}(\mathrm{n})$ and located around the central frequency $\left(\omega_{\mathrm{i}}-\omega_{0}\right)$ and an AWGN process $w(n)$ with power $\sigma_{w}{ }^{2}$.

$$
x(n)=d(n)+i(n) \cdot e^{j\left(\omega_{i}-\omega_{0}\right) n}+w(n)
$$

The large number of different carrier combinations makes the selection of the wanted carrier and of the interference difficult. It is evident that a MMSE design of the filter coefficients for a given combination of carriers for $d(n)$ and $i(n)$ does not yield an 
optimum solution (in the sense of the MMSE criterion) for another combination: of carriers. Indeed, if for instance the input that supplies the filter $h_{0}(n)$ is examined (Fig. 3.2), the $h_{0}(n)$ filter is wanted to have a constant gain response in the band of the carrier of interest, a null response in the band of the interfering carriers and a transilion band as narrow as possible (ideally, an instantaneous transition band).

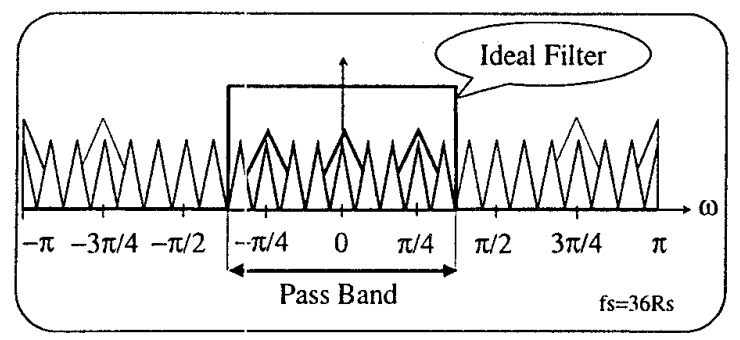

Figure 3.2. Ideal frequency response of ELP filter $h_{0}(n)$.

As will be seen later, the wanted signal and the interference are selected in order to force the MMSE design to provide a filter response with the desired spectral shape in the pass band, in the rejection band and in the transition band. Thus, although the actual signal used in the transmision is a cyclostationary signal (QPSK modulation), the signals involved in the model (Eq. 3.1) are assumed stationary and non-correlated.

\subsection{The MMSE Solution}

The ELP error signal depends on the impulse response of the filter with length $L$ as follows,

$$
e(n)=d(n)-\sum_{l=-(L-l) / 2}^{(L-1) / 2} \cdot 2(n-l) \cdot h(l) \equiv d(n)-h^{H} \cdot x_{n}
$$

with $\mathbf{h}^{\mathbf{H}}$ the filter coefficient vector and $\mathbf{x}_{\mathbf{n}}$ the input signal vector. Thus, the MMSE criterion minimizes the mean squared value of the decimated error (Eq. 3.2) with respect to the coefficients,

$$
\begin{aligned}
\xi= & E\left[\left|e\left(n^{\prime}\right)\right|^{2}\right]=E\left[\left|d\left(n^{\prime}\right)\right|^{2}\right]-E\left[d\left(n^{\prime}\right) \cdot x_{n^{\prime}}^{H}\right] \cdot h- \\
& -h^{H} \cdot E\left[d^{*}\left(n^{\prime}\right) \cdot x_{n^{\prime}}\right]+h^{H} \cdot E\left[x_{n^{*}} \cdot x_{n^{\prime}}^{H}\right] \cdot h
\end{aligned}
$$

leading to the well-known Wiener solution

$$
\boldsymbol{h}_{O P T}=\boldsymbol{R}_{x x}^{-1} \cdot \boldsymbol{r}_{x d}
$$

with $\mathbf{R}_{\mathrm{xx}}$ the autocorrelation matrix (Eq. 3.5) and $\mathbf{r}_{\mathrm{xd}}$ the crosscorrelation vector (Ec. 3.6).

$$
\begin{aligned}
& R_{x x}=E\left[x_{n^{\prime}} \cdot x_{n^{\prime}}^{H}\right] \equiv E\left[x_{n^{\prime} N} \cdot x_{n^{\prime} N}^{H}\right] \\
& r_{x d}=E\left[d^{*}\left(n^{\prime}\right) \cdot x_{n^{\prime}}\right] \equiv E\left[d^{*}\left(n^{\prime} N\right) \cdot x_{n^{\prime} N}\right]
\end{aligned}
$$

Assuming stationarity on the reference signal and on the interference, whose respective autocorrelation functions are denoted $r_{d d}(m)$ and $r_{i i}(m)$, it can be easily seen that the components of the autocorrelation matrix (Eq. 3.7) and of the cross-correlation vector (Eq.3.8),

$$
\begin{aligned}
& R_{x x}(m . l)=r_{x x}(m-l)= \\
& =r_{d d}(m-l)+r_{i i}(m-l) e^{j\left(\omega_{i}-\omega_{0}\right)(m-l)}+\sigma_{w}^{2} \cdot \delta(m-l) \\
& r_{x d}(m)=r_{d d}\left(m-1-\frac{L-1}{2}\right)
\end{aligned}
$$

lead to a Hermitian coefficient vector. Moreover, if the autocorrelation functions of the wanted and interfering signals are real and the difference between the central frequencies $\left(\omega_{i}-\right.$ $\left.\omega_{10}\right)$ is $\pi$, the ELP filter coefficients become real with even symmetry. This result is of great interest concerning a low cost VLSI implementation of those filters. Although it is out of the scope of this paper, it should be remarked that additional constraints on the filter coefficients can be imposed in the MMSE solution (Eq. 3.4) by means of the Lagrange multiplier method.

\subsection{Signal Model}

Concerning the selection of proper autocorrelation functions for the wanted signal and the interference, it should be pointed out that the frequency response of the resulting filter will depend on these functions. Thus, for a given input signal spectrum, denoted $S_{x x}(\omega)$, the frequency response of the filter will try to be one so that the output spectrum $S_{y y}(\omega)$ matches, as much as possible, the reference spectrum $S_{d d}(\omega)$.

The spectrum that models the reference and the interference for the case of the $h_{0}(n)$ is shown in Fig. 3.3.

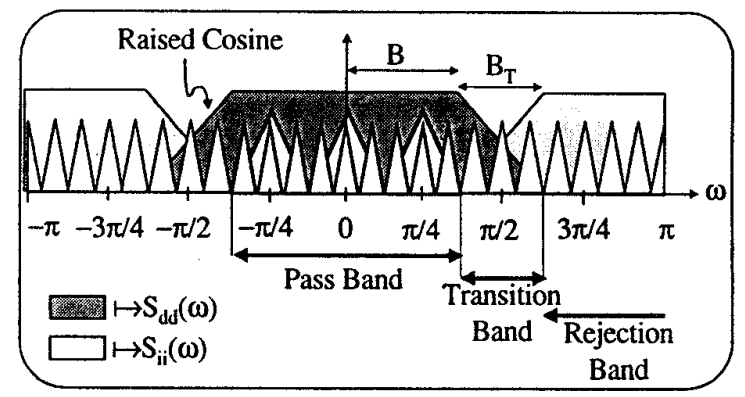

Figure 3.3. Spectrums for the reference, $S_{d d}(\omega)$ and the interference signals, $S_{\mathrm{ii}}(\omega)$.

The resulting filter will try to be as flat as possible in the band of interest, and as low and flat as possible in the interference band. In order to minimize the ripple effect of the frequency filter response, the transition band is set to the maximum value that does not introduce aliasing in the band of interest after decimation. The residual carriers that remain will be removed later by the matched filter. Important to remark is the fact that the spectrum follows a raised cosine function in the transition band. The purpose of this is twofold. Firstly, the filter frequency response will try to match as much as possible the raised cosine response, leading to a half-band or third-band filters, which null one out of two or three coefficients, respectively. Secondly, it is well known that the raised cosine response provides a good trade-off between signal bandwidth and temporal duration, and this property will provide filters with shorter impulse responses. The ELP autocorrelation function that corresponds to the spectral 
shape of the wanted and the interfering signals (Fig. 3.3) is the following one,

$$
\begin{gathered}
r_{d d}(m) \\
\quad \text { or } \\
r_{i i}(m)
\end{gathered}=\left\{\begin{array}{cc}
\left(B+B_{T} / 2\right) / \pi & m=0 \\
\frac{\sin \left(\left(B+B_{T} / 2\right) m\right)}{\pi m} \frac{\cos \left(\beta B_{T} m / 2\right)}{1-\left(\beta B_{T} m / \pi\right)} & m \neq 0
\end{array}\right.
$$

which depends on three parameters: the normalized pass band $B$, the normalized transition band, $B_{T}$, and the roll-off factor, $\beta$. The values of these parameters used to design each filter are summarized in Table 3.I.

\begin{tabular}{|c|c|c|c|c|}
\hline hi(n) Filter & \multicolumn{2}{|c|}{ Reference } & \multicolumn{2}{c|}{ Interference } \\
\hline $\mathrm{i}$ & $\mathrm{B}$ & $\mathrm{B}_{\mathrm{T}}$ & $\mathrm{B}$ & $\mathrm{B}_{\mathrm{I}}$ \\
\hline 0 & $3 \pi / 8$ & $\pi / 4$ & $3 \pi / 8$ & $\pi / 4$ \\
\hline $1,4,5$ & $\pi / 4$ & $\pi / 2$ & $\pi / 4$ & $\pi / 2$ \\
\hline $2,3\left(^{*}\right)$ & $\pi / 6$ & $\pi / 3$ & $\pi / 2$ & $\pi / 3$ \\
\hline
\end{tabular}

Table 3.I. Parameters for the design of $\left(h_{i}(n), i=0, \ldots, 5\right\}$.

The filter length is 19 , a value that has been decided by evaluating the MSSE for different filter lengths and by checking the EbNo in the final design. Nevertheless, $h_{2}(n)$ and $h_{3}(n)$ turn out to be third-band filters (that is, only 13 coefficients of the filters are non-zero), and the rest half-band filters (only 10 coefficients are non-zero)

\section{SIMULATION RESULTS}

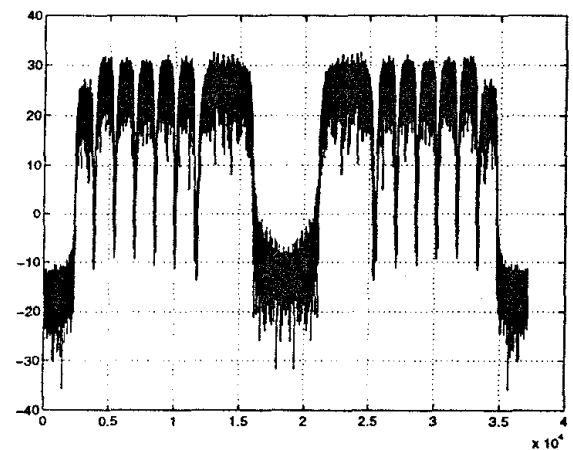

Figure 4.1. Spectral density for a 8-bit quantificated signal.

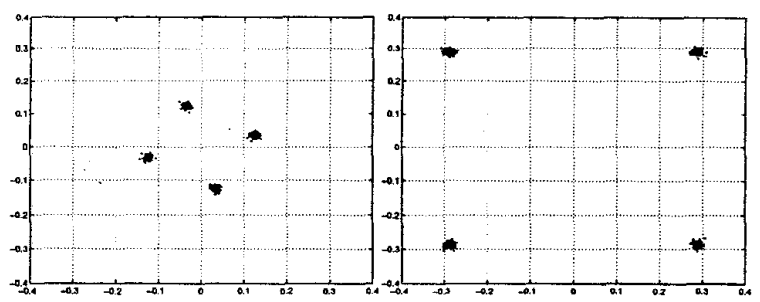

Figure 4.2. Constellations for one $2.1 \mathrm{Mbps}$ carrier with $\mathrm{Eb} / \mathrm{No}=21.1 \mathrm{~dB}$ (right) and $\mathrm{Eb} / \mathrm{No}=11.1 \mathrm{~dB}$ (left) respectively.

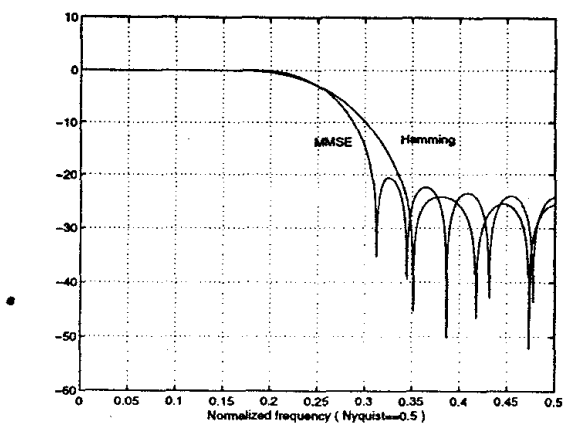

Figure 4.3. Filter design differences for the MMSE solution proposed and the Hamming window solution for the first stage of the 223 Tree Polyphase arquitecture.

\begin{tabular}{|c|c|c|}
\cline { 2 - 3 } \multicolumn{1}{c|}{} & Filter MMSE & Filter Hamming Window \\
\hline Channel 2.1 Mbps & 0.041 & 0.052 \\
\hline $\begin{array}{c}\text { Channel 2.1 Mbps } \\
\text { attenuated 10dB }\end{array}$ & 0.14 & 0.37 \\
\hline
\end{tabular}

Table 4.1. Design losses with a 223 Tree Polyphase arquitecture when $\mathrm{Eb} / \mathrm{No}=11 . \mathrm{I} \mathrm{dB}$.

\begin{tabular}{|c|c|c|}
\cline { 2 - 3 } \multicolumn{1}{c|}{} & Filter MMSE & Filter Hamming Window \\
\hline Channel 2.1 Mbps & 0.074 & 0.076 \\
\hline $\begin{array}{c}\text { Channel 2.1 Mbps } \\
\text { attenuated 10dB }\end{array}$ & 0.44 & 0.71 \\
\hline
\end{tabular}

Table 4.2. Implementation losses with a 223 Tree Polyphase arquitecture when $\mathrm{Eb} / \mathrm{No}=11.1 \mathrm{~dB}$.

\section{SUMMARY}

It has been shown that a suitable design criterion for the decimation filters in terms of a model of the interfering carriers can optimize to a great extent the ultimate complexity of a VLSI demultiplexing scheme. Conventional filter design methods of the same complexity are outperformed (see table 4.1-2), specially when the carrier of interest is attenuated. Incorporation of the knowledge of the interfering carrier spectrum helps optimize the transition and attenuation bands for the given filter length.

\section{REFERENCES}

11] J.Sala, A. Pagès, J.Riba, S.Calvo, G. Vázquez, M.A.Rey. "Algorithms Study and Simulation Results". Report AEO001887, 30/06/97 submitted to the European Space Agency under contract ESA/ESTEC-12092/96/NLUS.

[2] J.Prat, A. Rodríguez, F.Ortega, M.A.Rey. "Digital Architectural Design". Report AEO-0018487, 30/06/97 submitted to the European Space Agency under contract ESA/ESTEC-12092/96/NLUS

[3] F. Ortega, A. Rodriguez et al. "An advanced Multi-Carrier Demodulator for the ESA OBP System". Proceedings of the Fifth ESA International Workshop on Digital Signal Processing Techniques Applied to Space Communications. 